\title{
Cardiorespiratory effects of NIV-NAVA, NIPPV, and NCPAP shortly after extubation in extremely preterm infants: a randomized crossover trial
}

\author{
Samantha Latremouille ${ }^{1}$, Monica Bhuller ${ }^{1}$, Wissam Shalish ${ }^{1}$, and Guilherme Sant'Anna ${ }^{2}$ \\ ${ }^{1}$ McGill University Health Centre \\ ${ }^{2}$ Montreal Childrens Hospital
}

April 22, 2021

\begin{abstract}
Objective: Investigate the cardiorespiratory effects of non-invasive neurally adjusted ventilatory assist (NIV-NAVA), nonsynchronized nasal intermittent positive pressure ventilation (NIPPV), and nasal continuous positive airway pressure (NCPAP) during the critical period shortly after extubation. Hypothesis: Levels of non-invasive pressure support provided and/or presence of synchronization can affect cardiorespiratory parameters. Study design: Randomized crossover trial. Patient-subject selection: Infants with birth weight (BW) [?] 1250g undergoing their first planned extubation were randomly assigned to all 3 modes following extubation. Methodology: Electrocardiogram and electrical activity of the diaphragm (Edi) were recorded during 30min on each mode. Analysis of heart rate variability (HRV), diaphragmatic activity (Edi area, breath area, amplitude, inspiratory and expiratory times) and respiratory variability (RV) were compared between modes. Results: 23 enrolled infants had full data recordings and analysis: median $[\mathrm{IQR}]$ gestational age $=25.9$ weeks $[25.2-26.4]$, BW $=760 \mathrm{~g}[595-900]$, and post-natal age 7 [4-19] days. There were no differences in HRV parameters between modes. During NIV-NAVA and NIPPV, diaphragmatic activity was significantly lower and RV higher than NCPAP. Delivered peak inflation pressures (PIPs) were lower during NIV-NAVA than NIPPV $(14 \mathrm{cmH} 2 \mathrm{O}$ [13-16] vs cmH2O 16 [16-17]; p<0.001). However, due to a significantly higher proportion of assisted breaths (99\% [92-103] vs. 51\% [38-82]; p<0.001) NIV-NAVA provided a higher mean airway pressure (MAP) $(9.4 \mathrm{cmH} 2 \mathrm{O}$ [8.2-10.0] vs. $8.2 \mathrm{cmH} 2 \mathrm{O}$ [7.6-9.3]; $\mathrm{p}=0.002)$. Conclusions: NIV-NAVA and NIPPV applied shortly after extubation were associated with positive cardiorespiratory effects. This effect was more evident during NIV-NAVA where patient-ventilator synchronization provided a higher MAP with lower PIPs.
\end{abstract}

Title : "Cardiorespiratory effects of NIV-NAVA, NIPPV, and NCPAP shortly after extubation in extremely preterm infants: a randomized crossover trial"

Authors: Samantha Latremouille, MSc${ }^{1}$, Monica Bhuller, $\mathrm{MSc}^{1}$, Wissam Shalish, $\mathrm{MD}, \mathrm{PhD}^{2}$, Guilherme Sant'Anna, $\mathrm{MD}, \mathrm{PhD}^{3}$

Affiliations :

${ }^{1}$ Division of Experimental Medicine, McGill University Health Center; ${ }^{2}$ Assistant and ${ }^{3}$ Associate Professor of Pediatrics, Division of Neonatology, McGill University Health Center

Corresponding author:Guilherme Sant'Anna, MD, PhD, FRCPC Associate Professor of Pediatrics, Neonatal Division McGill University Health Center 1001 Boulevard Décarie, Room B05.2711 Montreal, Quebec, Canada. Zip Code: H4A3J1 Email: guilherme.santanna@mcgill.ca Phone: +1 (514) 4124452 extension 22389

Funding : This work was supported by the Montreal Children's Foundation research funds for G.S. In 
addition, S.L. and W.S. were both supported from Fonds de la recherche en sante du Quebec (FRQS) doctoral research awards in partnership with the Fondation Des Etoiles.

Word count: 3417

Key Words: Heart rate variability, Diaphragmatic activity, Respiratory variability, Non-invasive respiratory support

Running Head: Cardiorespiratory effects of non-invasive respiratory support

\section{ABSTRACT}

Objective: Investigate the cardiorespiratory effects of non-invasive neurally adjusted ventilatory assist (NIV-NAVA), non-synchronized nasal intermittent positive pressure ventilation (NIPPV), and nasal continuous positive airway pressure (NCPAP) during the critical period shortly after extubation.

Hypothesis: Levels of non-invasive pressure support provided and/or presence of synchronization can affect cardiorespiratory parameters.

Study design: Randomized crossover trial.

Patient-subject selection: Infants with birth weight (BW) [?] 1250g undergoing their first planned extubation were randomly assigned to all 3 modes following extubation.

Methodology: Electrocardiogram and electrical activity of the diaphragm (Edi) were recorded during 30min on each mode. Analysis of heart rate variability (HRV), diaphragmatic activity (Edi area, breath area, amplitude, inspiratory and expiratory times) and respiratory variability (RV) were compared between modes.

Results: 23 enrolled infants had full data recordings and analysis: median [IQR] gestational age $=25.9$ weeks [25.2-26.4], BW $=760 \mathrm{~g}$ [595-900], and post-natal age 7 [4-19] days. There were no differences in HRV parameters between modes. During NIV-NAVA and NIPPV, diaphragmatic activity was significantly lower and RV higher than NCPAP. Delivered peak inflation pressures (PIPs) were lower during NIV-NAVA than NIPPV $\left(14 \mathrm{cmH}_{2} \mathrm{O}\right.$ [13-16] vs $\mathrm{cmH}_{2} \mathrm{O} 16$ [16-17]; $\left.\mathrm{p}<0.001\right)$. However, due to a significantly higher proportion of assisted breaths $(99 \%$ [92-103] vs. $51 \%[38-82] ; \mathrm{p}<0.001)$ NIV-NAVA provided a higher mean airway pressure (MAP) $\left(9.4 \mathrm{cmH}_{2} \mathrm{O}[8.2-10.0]\right.$ vs. $\left.8.2 \mathrm{cmH}_{2} \mathrm{O}[7.6-9.3] ; \mathrm{p}=0.002\right)$.

Conclusions: NIV-NAVA and NIPPV applied shortly after extubation were associated with positive cardiorespiratory effects. This effect was more evident during NIV-NAVA where patient-ventilator synchronization provided a higher MAP with lower PIPs.

\section{INTRODUCTION}

Over the last decades neonatal intensive care has evolved dramatically, with a substantial increase in survival rates of extremely immature infants ${ }^{1}$. Amongst important interventions, a gentler respiratory care approach was paramount and supported by strong scientific evidence. The hallmark of such approach was the widespread adoption of non-invasive respiratory support as a primary strategy in an attempt to avoid mechanical ventilation (MV) and its associated complications ${ }^{2}$.

Nevertheless, extremely preterm infants still require MV during hospitalization, making optimized postextubation respiratory care $v_{i t a l}{ }^{1}$. A recent systematic review reinforced that preterm infants should be extubated to non-invasive respiratory support and that nasal continuous positive airway pressure (NCPAP) and non-synchronized non-invasive positive pressure ventilation (NIPPV) are the two most common modes ${ }^{3}$. Unfortunately, the certainty of the evidence to recommend one specific mode is downgraded due to risk of bias and imprecision of the included studies. As the negative effects of non-synchronized delivery of mechanical inflations have been previously described, including potentially harmful alterations in blood gases, blood pressure, and tidal volumes ${ }^{4}$, synchronization of NIPPV is desirable and has been shown to reduce inspiratory efforts $^{5}$. Regrettably, there are limited available methods for NIPPV synchronization ${ }^{6}$, thereby leading to 
suboptimal patient-ventilator interaction. Furthermore, the majority of studies investigated patients during a stable period, increasing the chances of hiding any beneficial effects.

Non-invasive neurally adjusted ventilatory assist (NIV-NAVA) is a mode of ventilation that uses the diaphragm electrical activity (Edi) signal to deliver synchronized mechanical inflations, thus improving patientventilator interaction ${ }^{7}$. NIV-NAVA is not affected by system leaks, provides continuous monitoring of respiratory pattern and drive, and has been successfully applied after extubation by some centers ${ }^{8}$. Analysis of cardiorespiratory behavior, including heart rate variability (HRV), diaphragmatic activity as surrogate of respiratory effort, and respiratory variability (RV), has been valuable in providing insight on the individualized physiological impact of different systems ${ }^{9-13}$. Thus, the objective of this randomized crossover study was to evaluate the effects of NIV-NAVA applied shortly after extubation in extremely preterm infants when compared to NIPPV and NCPAP by using analyses of cardiorespiratory behavior.

\section{METHODS}

\section{Population}

This prospective, unblinded, randomized crossover trial (ClinicalTrials.gov ID: NCT02723123) was conducted at the Montreal Children's Hospital neonatal intensive care unit (NICU) from July 2016 to August 2018. Patients with birth weight (BW) [?] 1250g, receiving MV and undergoing their first elective extubation attempt were eligible. Infants with major congenital anomalies, congenital heart defects, neuromuscular disease, diaphragmatic paralysis or palsy, diagnosed phrenic nerve injury, esophageal perforation, hemodynamic instability, or receiving narcotics or sedative agents were excluded. The institution's research ethics board approved the study and informed consent was obtained from parents or legal guardians.

Decisions related to extubation timing, post-extubation respiratory support provided outside the study period, and reintubation, were exclusively made by the treating team. As part of standard of care, all infants were extubated to either NCPAP or NIPPV and maintained at oxygen saturation $\left(\mathrm{SpO}_{2}\right)$ targets between $88-92 \%$.

\section{Study design and data acquisition}

Before extubation, 3 electrocardiogram (ECG) leads were placed on the infants' limbs, located at least $1 \mathrm{~cm}$ apart from the existing leads to prevent interference. Additionally, a 6 -French $/ 49 \mathrm{~cm}$ specialized feeding tube (NAVA catheter) used for the NAVA module of the Servo-i ventilator (Maquet Critical Care, Solna, Sweden) was placed at the level of the diaphragm and the position was confirmed by the Servo-i ventilator, as previously described ${ }^{14}$. After extubation, a brief period of stabilization (30-60 minutes) was allowed prior to initiation of recordings, during which the infants received the type of non-invasive respiratory support prescribed by the treating team. Following the stabilization period, infants received the study interventions (NIV-NAVA, NCPAP and NIPPV) applied in a computer-generated blocked random sequence generated created by author GS and implemented by SL, MB, and WS, with no allocation concealment. Infants received each randomised mode for 40 minutes, with the first 10 minutes considered as a wash-out period, where data were not used for analysis. A timeline of the study design is provided in Figure $\mathbf{1}$.

All infants were studied in resting supine position. NIV-NAVA, NIPPV, and NCPAP modes were provided by the Servo-i ventilator (Maquet Critical Care, Solna, Sweden) using infant binasal prongs (Hudson RCI, Wayne, Pennsylvania). For all 3 modes, the positive end-expiratory pressures (PEEP) or NCPAP levels matched the post-extubation pressures determined by the attending physician during the stabilization period. To provide NIPPV, the nasal pressure control mode on the Servo-i ventilator was used, which delivers flowsynchronized inflations via an internal sensor. Peak inflation pressures (PIP) set to $10 \mathrm{cmH}_{2} \mathrm{O}$ above the PEEP at a rate of $20 \mathrm{ipm}$ (inflations per minute). Increases in PIP and rates were permitted only if deemed necessary by the research staff or attending physician for infant stability and any deviations were recorded. For NIV-NAVA, the NAVA levels were adjusted trying to match the PIP levels provided during NIPPV.

Throughout the study period, the occurrence of bradycardias (heart rate $<100 \mathrm{bpm}$ ) and desaturations $\left(\mathrm{SpO}_{2}<85 \%\right)$ detected by the hospital's monitoring system were manually recorded by the research team 
at the bedside since these are the signals the health care professionals would react to; events occurring during the wash-out periods were excluded. Also, any change on the fraction of inspired oxygen $\left(\mathrm{FiO}_{2}\right)$ was documented.

\section{Primary outcome}

The primary outcome of the study was to evaluate the cardiorespiratory effects of NIV-NAVA applied shortly after extubation in extremely preterm infants when compared to NIPPV and NCPAP. For that, analyses of HRV, diaphragm activity and RV were performed and compared between modes.

\section{Heart rate variability}

The ECG signals were amplified, anti-alias filtered and sampled at $1000 \mathrm{~Hz}$ using a portable data acquisition system (FE132 Bio Amp and PowerLab, ADInstruments, Colorado, U.S.A). Signals were stored in a research computer for offline analysis using the LabChart software (Version 8.1.4, ADInstruments). ECG signals were analyzed using an in-house developed program based on the Pan and Tompkins, and Kota QRS detection methods ${ }^{15,16}$ and a publicly available HRV analysis tool HRVAS, available from https://github.com/jramshur/HRVAS. Documentation of the algorithm and MATLAB (MathWorks, Massachusetts, U.S.A.) application are publicly available at http://hrvtoolkit.com. The analysis was performed on the full 30-minute segment.

The following time domain parameters were calculated: median NN interval; standard deviation of the $\mathrm{NN}$ intervals, i.e. the variation of intervals measured between consecutive sinus beats (SDNN); the standard deviation of the averages of NN intervals in all 5-minute segments of the recording (SDANN); the mean of the standard deviations of all $\mathrm{NN}$ intervals for all 5 -minute segments of the recording (SDNNi); the percentage of adjacent $\mathrm{NN}$ intervals differing by $>50 \mathrm{~ms}$ (pNN50); root mean square of successive differences of $\mathrm{NN}$ intervals (RMSSD); total number of all NN intervals divided by the height of the 32-bin histogram of all NN intervals (triangular index; HRVTi); and the baseline width of the minimum square difference triangular interpolation of the highest peak of the 32-bin histogram of all NN intervals (TINN). The following non-linear parameters were also obtained: sample entropy (SampEn; $\mathrm{r}=0.1, \mathrm{~m}=3$ ); detrended fluctuations analysis (DFA) scaling exponents alpha1 (short-term; 4-13 beats), and alpha2 (long-term; 14-100 beats); the standard deviation of intervals perpendicular to the identity line of the Poincaré plot (SD1) and the standard deviation of intervals along the identity line (SD2). For thefrequency domain the following parameters were calculated: TP: total power $(<0.4 \mathrm{~Hz}), \mathrm{VLF}$ : very low frequency $(<0.04 \mathrm{~Hz}), \mathrm{LF}$ : low frequency $(0.04-0.15 \mathrm{~Hz}), \mathrm{HF}$ : high frequency $(>0.15$ to $<0.4 \mathrm{~Hz})$, and $\mathrm{LF} / \mathrm{HF}$ ratio using the Welch periodogram ${ }^{17,18}$.

\section{Respiratory analysis}

Diaphragmatic activity: Edi signals sampled at $100 \mathrm{~Hz}$ were acquired and stored for offline analysis using the Servo Tracker software (Maquet Critical Care, Solna, Sweden). For each patient and mode, Edi signals were analyzed using the full 30-minute segments to obtain the minimum value of Edi as the resting tonic state (Edi min), the maximum value of Edi as the maximal inspiratory effort (Edi max), and overall assessment of respiratory effort by calculating the area under the entire signal (Edi area). A breath-by-breath analysis was used to calculate: a) breath area, i.e. area under the breath curve; b) breath amplitude, i.e. the minimum vertical distance that the signal descends on either side of the peak (peak-to-trough distance); c) breath width, i.e. the distance between the points to the left and right of the peak at half the amplitude; d) neural inspiratory time (NTi), the time from trough to peak; and e) neural expiratory time (NTe), the time from peak to trough. The median values of these parameters were used for comparisons. An example of Edi calculation is provided inE-Figure $\mathbf{1}^{19}$. All parameters were computed using an automated algorithm developed on MATLAB (R2017a, MathWorks, Natick, U.S.A.), using peak detection functions with minimum amplitude of $1 \mu \mathrm{V}$ and at least $0.5 \mathrm{~s}$ apart.

Respiratory variability : Breath-to-breath (BB) time intervals were obtained and the following measures of variability were computed for breath areas, $\mathrm{NTi}$, NTe, breath amplitudes, and widths: standard deviation (SD), coefficient of variation (CV), and the standard deviation of the successive differences (SDSD) ${ }^{19}$. 


\section{Clinical data}

Baseline patient demographics were collected: BW, gestational age, gender, 1-minute and 5-minute Apgar scores, weight at extubation, postnatal age and post-menstrual age at extubation, pre-extubation blood gas ( $\mathrm{pH}$, partial pressure of $\mathrm{CO}_{2}$, bicarbonate, base excess), ventilator settings (ventilation mode, PIP, PEEP, mean airway pressure (MAP), $\mathrm{FiO}_{2}$, and inflation rate), and post-study non-invasive respiratory support (mode, PIP, PEEP, and inflation rates). In order to evaluate the study settings, pressure waveforms were analyzed offline to determine the number of PIPs delivered (in total and per minute), the proportion of assisted breaths (total number of PIPs divided by the total number of neural breaths), the median PIP value, and MAP over the entire recording for NIPPV and NIV-NAVA.

\section{Sample size and statistical analysis}

A convenience sample size of 20 infants was chosen. Given our experience with biological signal acquisition and analysis shortly after extubation, we expected up to 20-25\% patient loss due to poor signal quality, technical issues, clinical instability, or need of immediate reintubation ${ }^{9,11}$. Thus, the goal was to enroll 30 patients. All clinical and main outcome (HRV, diaphragmatic activity, RV) variables are expressed as median [IQR] or number (\%) and compared using the non-parametric Friedman's test with Tukey-Kramer's post-hoc test, Wilcoxon rank sum test, or Chi-square or Fisher's exact tests. Additionally, main outcome measures of NIV-NAVA and NIPPV were calculated and expressed as delta differences from NCPAP, which was considered the reference. Statistics were computed using MATLAB (R2018b, MathWorks, Massachusetts, U.S.A.) and p-value [?] 0.05 was considered significant.

\section{RESULTS}

A total of 30 infants were enrolled and studied, of which $23(77 \%)$ had full data recordings available for analysis; a flow diagram of patient recruitment is provided in E-Figure $\mathbf{2}$. These 23 infants had a median [IQR] gestational age of 25.9 weeks [25.2 - 26.4], BW of $760 \mathrm{~g}$ [595 - 900], and were studied at day 7 [4 - 19] of life (Table 1 ). Most infants were extubated from assist-control ventilation (96\%) and placed on NCPAP after extubation (70\%) (Table 1 ). Fourteen infants were successfully extubated while 9 failed, yielding a $39 \%$ reintubation rate within 7 days post-extubation.

During the study period, the median PEEP or NCPAP level was 7 [6 - 8] $\mathrm{cmH}_{2} \mathrm{O}$, PIP 17 [16-18] $\mathrm{cmH}_{2} \mathrm{O}$, rate 20 [20 - 20] ipm during NIPPV, and NAVA levels 1.0 [0.9 - 1.2] (Table 2 ). Setting changes were made in 3 infants based on clinical judgment: 1) NCPAP level increased from 7 to $8 \mathrm{cmH}_{2} \mathrm{O}, 2$ ) PIP/PEEP changed to 20/8 and rate of $40 \mathrm{ipm}$ during NIPPV, 3) rate increased to $40 \mathrm{ipm}$ during NIPPV. There were no significant differences in the $\mathrm{FiO}_{2}$ provided or presence of bradycardia/desaturation events between modes (Table 2 ).

Analysis of the pressure waveforms revealed median PIP delivered during NIV-NAVA of 14 [13 - 16] $\mathrm{cmH}_{2} \mathrm{O}$ compared to 16 [16 - 17] $\mathrm{cmH}_{2} \mathrm{O}$ during NIPPV $(\mathrm{p}<0.001)$. However, significantly more PIPs were delivered during NIV-NAVA (1375 [1155 - 1518] vs. 687 [649 - 731]; p<0.001), corresponding to a delivered inflation rate of $46 \mathrm{ipm}[38-51]$ vs. $23 \mathrm{ipm}[22-24](\mathrm{p}<0.001)$ and proportion of assisted breaths at $99 \%$ [92 - 103] vs. $51 \%[38-82](\mathrm{p}<0.001)$ during NIV-NAVA and NIPPV, respectively. This yielded a higher MAP during NIV-NAVA $\left(9.4 \mathrm{cmH}_{2} \mathrm{O}[8.2-10.0]\right.$ vs. $8.2 \mathrm{cmH}_{2} \mathrm{O}[7.6$ - 9.3]; $\mathrm{p}=0.002)($ Table 2$)$. An example of pressure and Edi waveforms during NIPPV and NIV-NAVA is provided as E-Figure 3 .

\section{Cardiorespiratory behavior}

$H R V$ : There were no significant differences observed for any HRV parameters between the three modes of support (E-Table 1 ).

Diaphragmatic activity : Significant changes were observed for diaphragmatic breath area, breath amplitude, and Edi area (Table 3 ) between modes. When compared to NCPAP, breath area was significantly lower during NIV-NAVA, and breath amplitude and Edi area were significantly lower during NIV-NAVA and NIPPV (Figure 2 ). 
$R V$ : There were significant differences across the 3 modes of support for RV measures of breath area, amplitude, and width (Table 4; Figure 2 ). A higher RV for all those measures was observed during NIVNAVA when compared to NCPAP. Specifically, variability of the breath area (CV) was significantly higher during NIV-NAVA compared to both NCPAP and NIPPV (Figure 2 ), and variability of both breath amplitude (CV) and width (SD, CV, and SDSD) were significantly higher during NIV-NAVA and NIPPV (Table 4; Figure 2 ) compared to NCPAP.

\section{DISCUSSION}

In this prospective crossover randomized trial, the cardiorespiratory effects of NIV-NAVA were compared to NCPAP and NIPPV in a high-risk population and during the unstable period shortly after extubation. Using comprehensive analyses of cardiorespiratory signals, NIV-NAVA was associated with decreased neural respiratory effort and increased RV when compared to ventilator-provided NCPAP. Moreover, these effects were more evident during NIV-NAVA where patient-ventilator synchronization provided a higher MAP with lower PIPs.

\section{Heart Rate Variability}

Respiration is well known to influence HRV through respiratory sinus arrhythmia (RSA), generating high frequency heart rate changes ${ }^{18}$. Studies in animals and adults have also shown that the application of continuous distending pressure leads to adaptive changes in autonomic cardiorespiratory control and alters HRV $^{20-22}$. Furthermore, measurements of HRV have been able to identify system instabilities before any clinical signs of deterioration, as demonstrated for the prediction of sepsis ${ }^{23}$, weaning from $\mathrm{MV}^{9}$ and outcomes of hypoxic-ischemic encephalopathy ${ }^{24}$. However, no significant differences in HRV measurements were found between NIV-NAVA, NCPAP, and NIPPV. These results are consistent with other studies comparing HRV between different non-invasive respiratory support modes in this population ${ }^{11,13}$. Evidently, since this has been a constant finding, differences in physiological effects between these modes are not reflected by changes in HRV during a short period of exposure.

\section{Diaphragmatic Activity}

Analysis of the diaphragmatic activity and pressure waveforms during NIV-NAVA and NIPPV yielded some interesting information. During NIPPV, the level of support received was different from that adjusted on the Servo-I, as the PIPs delivered were slightly but significantly lower. These PIPs however were greater than those delivered during NIV-NAVA, despite adjustments of the NAVA level in an attempt to match the PIPs at the beginning of the recording period. Interestingly, although lower PIP levels were provided during NIV-NAVA, the near-perfect synchronization between patient and ventilator on this mode yielded a higher number of delivered PIPs (E-Figure 3 ) and thus provided a higher MAP ${ }^{7}$. In that respect, infants seemingly adapt and reduce their respiratory efforts during NIV-NAVA. Indeed, a similar decrease has been demonstrated in preterm infants during other crossover studies comparing NCPAP, biphasic positive airway pressure or NIPPV with NIV-NAVA ${ }^{12,25,26}$.

A decreased neural respiratory effort was observed during NIV-NAVA and NIPPV when compared to NCPAP. The higher MAP provided during NIPPV (median $=8.2$ ) and NIV-NAVA (median $=9.3$ ) proportionally offloaded the diaphragm, taking some of the work of breathing away from the infant as previously described $8,12,26$. Interestingly, the NIPPV mode on the Servo-i ventilator has flow-synchronization using an internal sensor, which this study has demonstrated to be largely ineffective. Indeed, only $51 \%$ of the infant's spontaneous breaths were assisted during NIPPV. This was clearly noted upon inspection of the ventilator pressures and Edi waveforms (E-Figure 3 ) as previously demonstrated ${ }^{7}$.

\section{Respiratory Variability}

Breathing is a variable physiological process that represents the continuous adaptation of the respiratory control systems to maintain homeostasis. As such, a certain amount of variability in breathing is normal: a continuously adjusting balance between consistency and complexity. Indeed, changes in RV have been demonstrated in response to changes in mechanical loading ${ }^{27-29}$, respiratory morbidities ${ }^{30,31}$ and extubation 
outcomes $^{10}$, and has been compared between modes of respiratory support ${ }^{27,32}$. Both reduced ${ }^{27,32}$ and increased RV ${ }^{33,34}$ have been associated with clinical deterioration and worse outcomes. Thus, changes in RV should be interpreted with caution and must consider the context.

A higher RV in infants receiving invasive NAVA ventilation has been previously observed, though the differences did not reach statistical significance ${ }^{27}$. Although our study investigated non-invasive modes, an increased RV was also noted during NIV-NAVA when compared to NIPPV and NCPAP. If the RV calculated during NCPAP is the normal baseline, an increase could be due to erratic and unstable breathing patterns during NIV-NAVA ${ }^{33,34}$. But if the NCPAP values reflect a reduced RV from normal baseline, then NIV-NAVA may be conferring a beneficial effect. Indeed, during MV, decreases in mechanical loads have been associated with increases on $\mathrm{RV}^{28,29}$. Furthermore the increased RV observed during NIV-NAVA when compared to NIPPV and NCPAP may be a reflection of the additional synchronized PIPs from the ventilator. Actually, as the increased variability occurred for both neural breathing depth (breath area and amplitude) and timing (breath width), it is likely that this is related to the effective transmission of the pressures from the ventilator 27,29 and therefore may be regarded as a beneficial physiological response to this mode of non-invasive support. It remains unclear if these findings would persist if each mode were provided at exactly the same MAP or for longer periods. Future studies of non-invasive modes of respiratory support should consider matching MAP (if possible) to compare their influence on RV and longer recording periods.

A few issues were noted with the use of NIV-NAVA. First, maintaining proper catheter placement was not always a simple task and given the extremely small size of our population, a small displacement could negatively impact the Edi signal quality and pressure delivery. Second, NIV-NAVA only works well in infants with a good respiratory drive. Indeed, the 3 infants that failed NCPAP during recordings also had difficulties during NIV-NAVA. A poor respiratory drive elicited low PIPs due to the proportionality of the NAVA algorithm. Also, we noticed that the back-up ventilation turned on and off in repeated cycles, which did not allow for proper back-up support and stabilization. The apnea detection time used to trigger backup ventilation was of 10 s and a shorter period may have been more appropriate in these patients ${ }^{35}$. While some small randomized controlled trials ${ }^{8,36}$ and retrospective studies ${ }^{37-39}$ reported reduced respiratory failure rates with NIV-NAVA, there is a lack of good evidence to determine its superiority over other modes of noninvasive support ${ }^{40}$. Thus, until larger randomized trials are conducted, some caution should be exercised when integrating NIV-NAVA into the care of extremely preterm infants.

\section{Limitations \& Strengths}

This study has some limitations. We used relatively short observation windows on each mode, and it is unclear how the results may have changed with longer exposure times. The presence of desaturations and bradycardia events were manually recorded as free text, lacking specific information on the frequency and duration of these events. We used ventilator instead of bubble CPAP to prevent multiple changes of devices during the study. Thus, physiological differences might be different when comparing to the bubble system. However, the study has many important strengths. The randomized crossover design allowed each patient to act as their own control, which is of particular importance in physiological studies due to strong interindividual variability. To date, this is the largest randomized crossover study of NIV-NAVA in extremely preterm infants, with only one small crossover study performed during the immediate extubation period ${ }^{12}$. This is also the only crossover study comparing NIV-NAVA with 2 other modes of non-invasive respiratory support and performing analyses of cardiac and respiratory variability. Furthermore, HRV was analyzed and calculated following the Task Force recommendations.

\section{CONCLUSION}

In a cohort of extremely preterm infants, the use of NIV-NAVA and NIPPV shortly after extubation was associated with decreased neural respiratory effort and increased RV when compared to ventilator-provided NCPAP. This effect was more evident during NIV-NAVA where patient-ventilator synchronization provided a higher MAP despite lower PIP levels. 


\section{Acknowledgement}

We would like to thank Smita Rao (research assistant) for her help with patient recruitment and data collection. We are indebted to all infants, their families, and the NICU staff for their participation and help with the study.

\section{Disclosures: None}

\section{REFERENCES}

1. Stoll BJ, Hansen NI, Bell EF, Walsh MC, Carlo WA, Shankaran S, Laptook AR, Sánchez PJ, Van Meurs KP, Wyckoff M, et al. Trends in Care Practices, Morbidity, and Mortality of Extremely Preterm Neonates, 1993-2012. JAMA 2015;314(10):1039-1051.

2. Sant'Anna GM, Keszler M. Weaning infants from mechanical ventilation. Clin Perinatol 2012;39(3):543562.

3. Ferguson KN, Roberts CT, Manley BJ, Davis PG. Interventions to Improve Rates of Successful Extubation in Preterm Infants: A Systematic Review and Meta-analysis. JAMA Pediatr 2017;171(2):165-174.

4. Greenough A, Morley C, Davis J. Interaction of spontaneous respiration with artificial ventilation in preterm babies. J Pediatr 1983;103(5):769-773.

5. Chang H-Y, Claure N, D'Ugard C, Torres J, Nwajei P, Bancalari E. Effects of synchronization during nasal ventilation in clinically stable preterm infants. Pediatr Res 2011;69(1):84-89.

6. Cummings JJ, Polin RA. Noninvasive Respiratory Support. Pediatrics 2016;137(1).

7. Stein H, Beck J, Dunn M. Non-invasive ventilation with neurally adjusted ventilatory assist in newborns. Semin Fetal Neonatal Med 2016;21(3):154-161.

8. Makker K, Cortez J, Jha K, Shah S, Nandula P, Lowrie D, Smotherman C, Gautam S, Hudak ML. Comparison of extubation success using noninvasive positive pressure ventilation (NIPPV) versus noninvasive neurally adjusted ventilatory assist (NI-NAVA). J Perinatol 2020:1-9.

9. Kaczmarek J, Chawla S, Marchica C, Dwaihy M, Grundy L, Sant'Anna GM. Heart rate variability and extubation readiness in extremely preterm infants. Neonatology 2013;104(1):42-48.

10. Kaczmarek J, Kamlin CO, Morley CJ, Davis PG, Sant'anna GM. Variability of respiratory parameters and extubation readiness in ventilated neonates. Arch Dis Child Fetal Neonatal Ed 2013;98(1):F70-73.

11. Latremouille S, Al-Jabri A, Lamer P, Kanbar L, Shalish W, Kearney RE, Sant'Anna GM. Heart Rate Variability in Extremely Preterm Infants Receiving Nasal CPAP and Non-Synchronized Noninvasive Ventilation Immediately After Extubation. Respir Care 2018;63(1):62-69.

12. Lee J, Kim HS, Jung YH, Shin SH, Choi CW, Kim EK, Kim BI, Choi JH. Non-invasive neurally adjusted ventilatory assist in preterm infants: a randomised phase II crossover trial. Arch Dis Child Fetal Neonatal Ed 2015;100(6):F507-513.

13. Latremouille S, Shalish W, Kanbar L, Lamer P, Rao S, Kearney RE, Sant'Anna GM. The effects of nasal continuous positive airway pressure and high flow nasal cannula on heart rate variability in extremely preterm infants after extubation: A randomized crossover trial. Pediatr Pulmonol 2019;54(6):788-796.

14. Stein H, Hall R, Davis K, White DB. Electrical activity of the diaphragm (Edi) values and Edi catheter placement in non-ventilated preterm neonates. J Perinatol 2013;33(9):707-711.

15. Kota S, Swisher CB, Al-Shargabi T, Andescavage N, du Plessis A, Govindan RB. Identification of QRS complex in non-stationary electrocardiogram of sick infants. Comput Biol Med 2017;87:211-216.

16. Pan J, Tompkins WJ. A Real-Time QRS Detection Algorithm. IEEE Trans Biomed Eng 1985;BME$32(3): 230-236$. 
17. Sassi R, Cerutti S, Lombardi F, Malik M, Huikuri HV, Peng CK, Schmidt G, Yamamoto Y. Advances in heart rate variability signal analysis: joint position statement by the e-Cardiology ESC Working Group and the European Heart Rhythm Association co-endorsed by the Asia Pacific Heart Rhythm Society. Europace 2015;17(9):1341-1353.

18. Task Force of The European Society of Cardiology and The North American Society of Pacing and Electrophysiology. Heart rate variability: standards of measurement, physiological interpretation and clinical use. Circulation 1996;93(5):1043-1065.

19. Latremouille S, Bhuller M, Rao S, Shalish W, Sant'Anna G. Diaphragmatic activity and neural breathing variability during a 5 -min endotracheal continuous positive airway pressure trial in extremely preterm infants. Pediatr Res 2020:1-8.

20. Frazier SK, Moser DK, Stone KS. Heart rate variability and hemodynamic alterations in canines with normal cardiac function during exposure to pressure support, continuous positive airway pressure, and a combination of pressure support and continuous positive airway pressure. Biol Res Nurs 2001;2(3):167-174.

21. Looga R. Reflex cardiovascular responses to lung inflation: a review. Respir Physiol 1997;109(2):95-106.

22. Pantoni C, Di Thommazo L, Mendes R, Catai A, Luzzi S, Amaral Neto O, Borghi-Silva A. Effects of different levels of positive airway pressure on breathing pattern and heart rate variability after coronary artery bypass grafting surgery. Braz J Med Biol Res 2011;44(1):38-45.

23. Fairchild KD, Schelonka RL, Kaufman DA, Carlo WA, Kattwinkel J, Porcelli PJ, Navarrete CT, Bancalari E, Aschner JL, Walker MW. Septicemia mortality reduction in neonates in a heart rate characteristics monitoring trial. Pediatr Res 2013;74(5):570-575.

24. Goulding RM, Stevenson NJ, Murray DM, Livingstone V, Filan PM, Boylan GB. Heart rate variability in hypoxic ischemic encephalopathy: correlation with EEG grade and 2-y neurodevelopmental outcome. Pediatr Res 2015;77(5):681-687.

25. Gupta A, Lumba R, Bailey S, Verma S, Patil U, Mally P. Electrical Activity of the Diaphragm in a Small Cohort of Preterm Infants on Noninvasive Neurally Adjusted Ventilatory Assist and Continuous Positive Airway Pressure: A Prospective Comparative Pilot Study. Cureus 2019;11(12):e6291.

26. Gibu CK, Cheng PY, Ward RJ, Castro B, Heldt GP. Feasibility and physiological effects of noninvasive neurally adjusted ventilatory assist in preterm infants. Pediatr Res 2017;82(4):650-657.

27. Baudin F, Wu H-T, Bordessoule A, Beck J, Jouvet P, Frasch MG, Emeriaud G. Impact of ventilatory modes on the breathing variability in mechanically ventilated infants. Front Pediatr 2014;2:132.

28. Brack T, Jubran A, Tobin MJ. Effect of Resistive Loading on Variational Activity of Breathing. Am J Respir Crit Care Med 1998;157(6):1756-1763.

29. Schmidt M, Demoule A, Cracco C, Gharbi A, Fiamma MN, Straus C, Duguet A, Gottfried SB, Similowski T. Neurally adjusted ventilatory assist increases respiratory variability and complexity in acute respiratory failure. Anesthesiology 2010;112(3):670-681.

30. Hutten GJ, van Eykern LA, Latzin P, Thamrin C, van Aalderen WM, Frey U. Respiratory muscle activity related to flow and lung volume in preterm infants compared with term infants. Pediatr Res 2010;68(4):339.

31. Usemann J, Suter A, Zannin E, Proietti E, Fouzas S, Schulzke S, Latzin P, Frey U, Fuchs O, Korten I, et al. Variability of Tidal Breathing Parameters in Preterm Infants and Associations with Respiratory Morbidity during Infancy: A Cohort Study. J Pediatr 2019;205:61-69.e61.

32. de Waal C, Hutten G, Kraaijenga J, de Jongh F, van Kaam A. Electrical activity of the diaphragm during nCPAP and high flow nasal cannula. Arch Dis Child Fetal Neonatal Ed 2017;102(5):F434-F438. 
33. Navarro X, Porée F, Beuchée A, Carrault G. Artifact rejection and cycle detection in immature breathing: Application to the early detection of neonatal sepsis. Biomed Signal Process Control 2015;16:9-16.

34. Patel M, Mohr M, Lake D, Delos J, Moorman JR, Sinkin RA, Kattwinkel J, Fairchild K. Clinical associations with immature breathing in preterm infants: part 2 - periodic breathing. Pediatr Res 2016;80(1):28-34.

35. Stein H, Firestone K. NAVA ventilation in neonates: clinical guidelines and management strategies. Neonatol Today 2012;7(4):1-8.

36. Kallio M, Mahlman M, Koskela U, Aikio O, Suo-Palosaari M, Pokka T, Saarela T, Hallman M. NIV NAVA versus Nasal CPAP in Premature Infants: A Randomized Clinical Trial. Neonatology 2019;116(4):380-384.

37. Lee BK, Shin SH, Jung YH, Kim EK, Kim HS. Comparison of NIV-NAVA and NCPAP in facilitating extubation for very preterm infants. BMC Pediatr 2019;19(1):298.

38. Yagui ACZ, Gonçalves PA, Murakami SH, Santos AZ, Zacharias RSB, Rebello CM. Is noninvasive neurally adjusted ventilatory assistance (NIV-NAVA) an alternative to NCPAP in preventing extubation failure in preterm infants? J Matern Fetal Neonatal Med 2019:1-151.

39. Yonehara K, Ogawa R, Kamei Y, Oda A, Kokubo M, Hiroma T, Nakamura T. Non-invasive neurally adjusted ventilatory assist versus nasal intermittent positive-pressure ventilation in preterm infants born before 30 weeks' gestation. Pediatr Int 2018;60(10):957-961.

40. Goel D, Oei JL, Smyth J, Schindler T. Diaphragm-triggered non-invasive respiratory support in preterm infants. Cochrane Database Syst Rev 2020;3(3):Cd012935.

\section{Hosted file}

NIV-NAVA_ImageLegends.pdf available at https://authorea.com/users/409439/articles/519124cardiorespiratory-effects-of-niv-nava-nippv-and-ncpap-shortly-after-extubation-inextremely-preterm-infants-a-randomized-crossover-trial

\section{Hosted file}

NIV-NAVA_Tables.pdf available at https://authorea.com/users/409439/articles/519124cardiorespiratory-effects-of-niv-nava-nippv-and-ncpap-shortly-after-extubation-inextremely-preterm-infants-a-randomized-crossover-trial

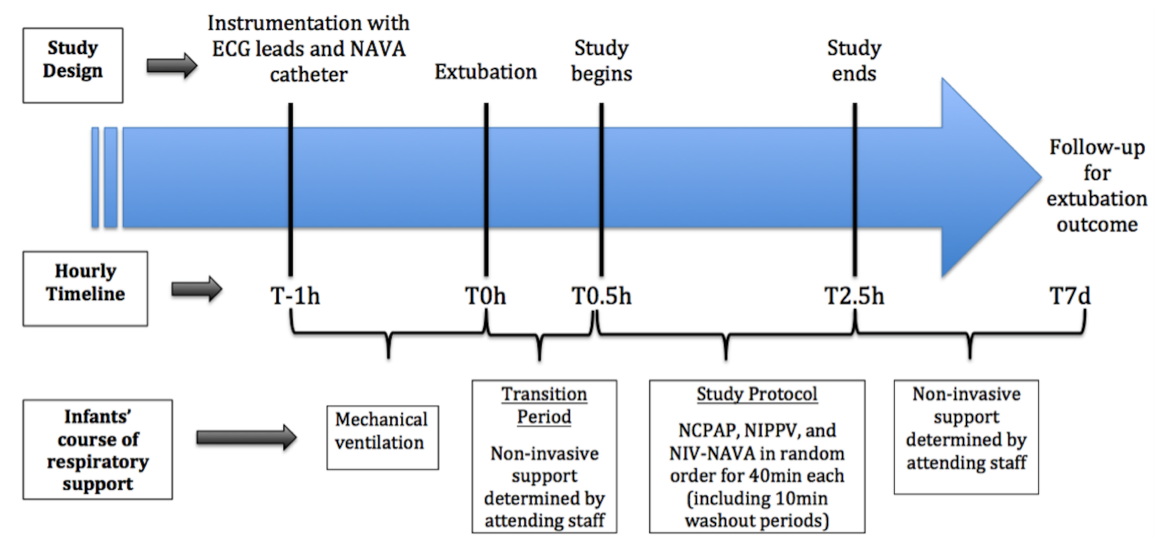



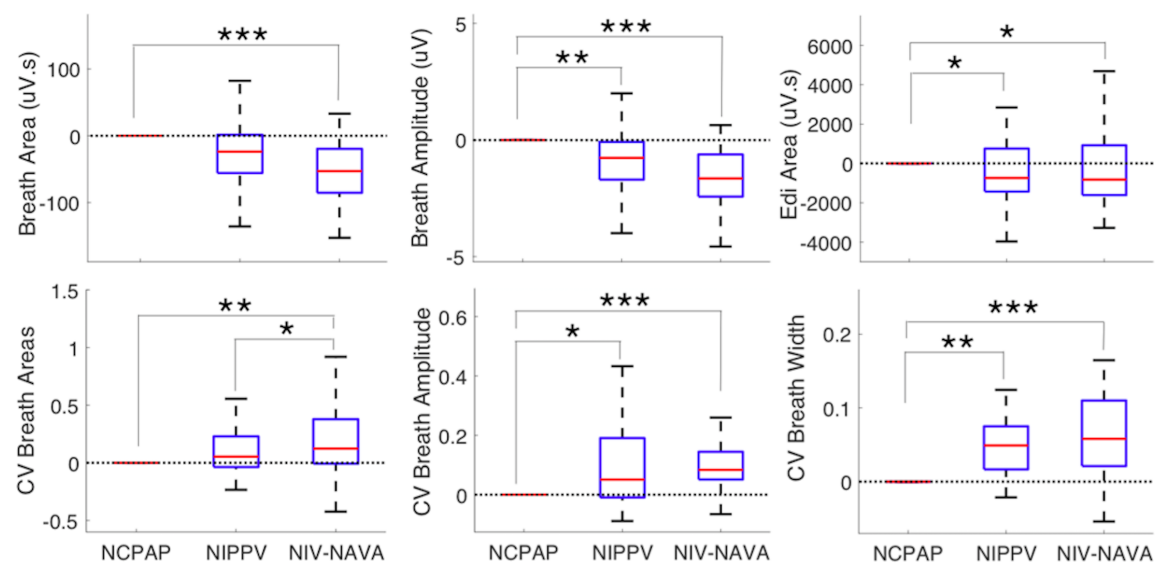